\title{
Valid Geometric Solutions for Indentations with Algebraic Calculations
}

\author{
Gerd Kaupp \\ University of Oldenburg, Oldenburg, Germany \\ Email: gerd.kaupp@uni-oldenburg.de
}

How to cite this paper: Kaupp, G. (2020) Valid Geometric Solutions for Indentations with Algebraic Calculations. Advances in Pure Mathematics, 10, 322-336.

https://doi.org/10.4236/apm.2020.105019

Received: March 9, 2020

Accepted: May 18, 2020

Published: May 21, 2020

Copyright $\odot 2020$ by author(s) and Scientific Research Publishing Inc. This work is licensed under the Creative Commons Attribution International License (CC BY 4.0).

http://creativecommons.org/licenses/by/4.0/

\begin{abstract}
This paper analyzes the force vs depth loading curves of conical, pyramidal, wedged and for spherical indentations on a strict mathematical basis by explicit use of the indenter geometries rather than on still world-wide used iterated "contact depths" with elastic theory and violation of the energy law. The now correctly analyzed loading curves provide as yet undetectable phasetransition. For the spherical indentations, this includes an obvious correction for the varying depth/radius ratio, which had previously been disregarded. Only algebraic formulas are now used for the calculation of material's properties without data-fittings, or simplifications, or false simulations. Penetration resistance differences of materials' polymorphs provide precise intersection values as kink unsteadiness by equalization of linear regression lines from mathematically linearized loading curves. These intersections indicate phase transition onset values for depth and force. The precise and correct determination of phase-transition onsets allows for energy and phase-transition energy calculations. The unprecedented algebraic equations are most simply and mathematically reproducibly deduced. There are no restrictions for elastic and/or plastic behavior and no use of different formulas for different force ranges. The novel indentation formulas reveal unprecedented access to the onset, energy and transition energy of phase-transitions. This is now also achieved for spherical indentations. Their formula as deduced for plotting is reformulated for integrations. The distinction of applied work $\left(W_{\text {applied }}\right)$ and indentation work $\left(W_{\text {indent }}\right)$ allows now for comparing spherical with pyramidal indentation phase-transitions. Only low energy phase-transitions from pyramidal indentation may be missed in spherical indentations. The rather low penetration depths of sphere calottes calculate very close for cap and flat area values. This allows for the calculation of the indentation phase-transition onset pressure and thus the successful comparison with hydrostatic anvil pressurizing results. This is very helpful for their interpretations, as low energy phase-transitions are often missed under the anvil, and it further strengthens
\end{abstract}


the unparalleled ease of the indentation techniques. Exemplification is reported for pyramidal, spherical, and hydrostatic anvil stressing by the numerical analysis of published germanium data. The previous widely accepted historical indentation theories and standards are challenged. Falsely simulated and even published so-called "experimental" indentation data from the literature can most easily be checked. They are mathematically unsound and their correction is urgently necessary for scientific reasons and daily safety with stressed materials. The motivation for this paper is the challenge of worldwide incorrect ISO 14577 standards for false and incomplete characterization of materials. The minimization of catastrophic failures e.g. in aviation requires the strengthening and the advancements of the mathematical truth by using our closed formulas that are based on undeniable geometric and algebraic calculation rules.

\section{Keywords}

Geometry of Indenters, Algebraic Solutions, False Mathematic Concepts, Germanium, Pyramidal Conical Indentation, Spherical Indentation

\section{Introduction}

Normal indentations onto flat surfaces are a long-term mathematical problem initially posed by Boussinesq in 1882 [1]. The load/depth relation had always been tried to be solved by starting with elasticity theory. Young's modulus $E$ (that is unfit for indentations) and Poisson's ratio $v$ unduly described the materials by indentations with the total applied force for an (since 1992 iterated) indentation area. The highly cited work of Hertz [2] in 1882 described only the mathematical touching between balls (with radius $R$ ) and the one of balls with flat surfaces, but without penetration. He deduced a contact pressure $p=k \alpha^{3 / 2}$ where $\alpha$ describes the impact area. He repeated that he did not describe penetration in [3], but all followers who refined this approach kept with a force $P$ per indentation area a (circle of contact) when the half-sphere penetrated at increasing force. They had to deal with an additional parameter the indentation depth $h$ and kept with the exponent $3 / 2$ on the penetration depth $\left(h^{3 / 2}\right)$. Much effort was put to the refinement in the early 1900ies, but advancements became over complicated, and also conical indentation had to be mathematically described. Really useful equations for practical use had to wait until 1939, when Love in [4] very laboriously deduced formulas for rigid cones. His approach was again "elastic theory" and force per area and his solution is $P=h^{2} \pi \operatorname{tg} \alpha E / 2\left(1-v^{2}\right)$ (below we replace $P$ by $F_{N}$ for normal force and do not violate the energy law). Thus, the depth was squared for cones and pyramids in [4]. Only the proportionality factor was changed in 1965 with the very laborious deduction of Sneddon in [5] to read $P=h^{2} \cdot 2 E \cot \alpha / \pi\left(1-v^{2}\right)$ that has been widely accepted. He also deduced a "solution" for spherical indentation by using Hankel transforms and the theory of dual integral equations. His formula (6.15) for spheres is 
$P=E(1-v)^{-1}\left\{\left(a^{2}+R^{2}\right) 2 h / a-a R\right\}$, where $a^{2}=R^{2}-(R-h)^{2}$. It at least shows that the situation is much more difficult than "Hertzian theory". When the substitution is made one obtains an equation $P=E(1-v)^{-1}\left(2 R h-h^{2}\right)^{-1 / 2}(5 R-2 h) h^{3 / 2}$ with a multitude of terms and with numerous different exponents on $h$. Only this part of Sneddon's work was ignored. 20 years later Johnson came back in [6] with a formula by "summarizing Hertzian theory" to read $P=4 / 3 E^{*} R^{1 / 2} h^{3 / 2}$ (where $E^{*}$ is reduced elastic modulus) for the sphere. This again did not consider the particular geometry of the sphere calotte. Interestingly, Oliver and Pharr cited in [7] the reference [5] but formulated " $P=\alpha h^{m}$ for spheres "with $m=1.5$ ", arguing that the sphere can be described as a solid of revolution giving "a smooth function". But they did not tell that this was Johnson's "summarized" formula in [6]. Unfortunately, the geometrically unsound formulas in [7] and in [6] became ISO-ASTM standard (International Standardization Organization-American Society for Testing of Materials) that is still generally enforced. For example, the authors of [8] calculated modulus values $E$ from the elastic contact with spherical indentation "according to the classical Hertzian theory" with Johnson's formula in [6] ("below the yield point"). It did not help that the experimental loading curves were at variance with these formulas. The exponents were just believed but not checked. Such checking was at least possible since 2004 as shown in [9] and thereafter with the $F_{N}$ vs $h^{3 / 2}$ plot from the present author's group. This plot (later from believers disdainfully termed as "Kaupp-plot") disproved and disproves the more than 1000-fold falsely claimed exponent " 2 " on $h$ (as enforced by ISO) for all conical, pyramidal and wedged indentations. It also discloses whether published spherical indents were truly spherical. However, several researchers continued to simulate spherical indentations as one exponent parabola with $h^{3 / 2}$ according to Johnson's formula in [6] and claimed that their "experimental curves" would support such claims. Fortunately, such published "results" of "spherical indentations" have been and can be disproved by exponent check with the $F_{N}$ vs $h^{3 / 2}$ plot (we now also call it "Kaupp-plot"), which, of course, cannot give straight lines neither for the simulation and nor for the published so called "experimental" curves. Such publications are disastrous and some examples for such clearly manipulated data are published in [10] [11]. We refrain from listing further examples; they are easily checked in the literature. The here cited disclosures and our papers with mathematical deductions on the basis of undeniable closed mathematical equations for indentations have not yet occasioned ISO-ASTM to thoroughly revise their incorrect ISO 14577 standards, falsely enforcing the producing industries via the certification agencies. We therefore extend our mathematical deductions for conical, pyramidal, wedged and spherical indentations and report various unprecedented application. This also allows for comparison of unchanged spherical indentations with hydrostatic techniques.

\section{The Geometrical Deductions of Indentation Formulas Excluding Iterations}

All mathematical deductions in the Introduction started with the indented sur- 
face area, and by applying the elasticity theory. They ran into enormous mathematical problems that could for a long time not be solved since 1882 with practically useable formulas. Only the still incorrect formulas of Love in [4] or one of them from Sneddon in [5] and of Johnson in [6] were used by Oliver-Pharr [7] in 1992 by using standard materials that they characterized by two iterations with 3 followed by 8 free parameters. These iterations were taken up by ISO and refined as ISO 14577 standards for performing and analyzing indentations. These physically unsound but binding standards had not been challenged before 2004 [9] when the present author's group started to empirically prove with the Kaupp-plot that such standards did experimentally not concur. It was undeniably deduced in 2013 [12] that the standards violated the energy law because not all applied force and energy is used for the volume formation. Finally, the mathematical foundation of the $F_{N}$ vs $h^{3 / 2}$ parabola for cones was geometrically deduced in 2016 using basic algebra in [13]. The projected or iterated contact area related formulas are thus finally disproved for conical, pyramidal and wedged indentations. Rather the volume of the indenter has to be used and everything is very simple. Previous thinking is thus obsolete. We must now comprehensibly repeat the geometrical deduction despite their simplicity, as the incorrect ISO 14577 standards are still used by teachers, and enforced to certification agencies, and thus also for the producing industries.

\subsection{The Energetics and the Correct Exponent of Conical Indentations}

The normal force vs depth curves are empirically described since 2004 in [9] and also theoretically since 2016 in [13] as parabolas with exponent $3 / 2$ on the depth as Formula (1). $F_{N}$ is the normal force (we do not use " $P$ " as in the formulas of the Introduction), $h(\mu \mathrm{m})$ is the depth, and $k\left(\mathrm{mN} / \mu \mathrm{m}^{3 / 2}\right)$ is the material's penetration resistance. The indentation work $W_{\text {indent }}(\mathrm{mN \mu m})$ in Formula (2) is obtained by integration. The constantly increased normal force from zero to the same force gives the applied work $W_{\text {applied }}(\mathrm{mN} \mu \mathrm{m})$ in Formula (3). For the maximal force $F_{N \max }$ we substitute $F_{N}$ of Formula (1) in Formula (3) and obtain the $W_{\text {applied }} / W_{\text {indent }}$ ratio of $5 / 4$, which is universally valid for all materials upon conical, pyramidal and wedged indentations. Clearly $20 \%$ of $W_{\text {applied }}$ (and thus $F_{N}$ ) is not used for the penetration with a cone, pyramid or wedge. The non-consideration is the already mentioned violation of the energy law that led to a false exponent on $h$, which is still enforced by ISO, but urgent subject to change. This is the reason why we use $F_{N}$ and not " $P$ " for the normal force.

$$
\begin{aligned}
F_{N} & =k h^{3 / 2} \\
W_{\text {indent }} & =0.4 k h^{5 / 2} \\
W_{\text {applied }} & =0.5 F_{N} h
\end{aligned}
$$

The geometric deduction of the correct exponent ( $3 / 2$ but not 2) in [13] has to consider that the penetration of the cone under force is a coupled process of vo- 
lume- and pressure-formation. In practice there is not always only elastic pressure but there are mostly all kinds of plastic deformations. We thus sum up all of it to "total pressure". It creates the $20 \%$ loss of force and energy for the volume formation with its depth. One has to multiply the force for volume formation $F_{N_{V}}$ with the force for total pressure formation $F_{N p}$ in Equation (4). Now one considers that the total pressure must be proportional to the immersed volume of the cone in Equation (5), so that $F_{N p}$ is proportional to $h^{3}$ and $h$ proportional to $F_{N p}^{1 / 3}$. When the exponent $\mathrm{n}$ is $1 / 3$ the exponent m must be $2 / 3$ and Equation (4) becomes Equation (6). As $F_{N p}^{1 / 3}$ is lost for the indentation only $F_{N v}^{2 / 3}$ is proportional to the depth in Equation (7). The deduction is completed with inclusion of the materials property factor, which is the penetration resistance or the physical hardness $k_{v}$ or $k=1.25 k_{v}\left(\mathrm{mN} / \mu \mathrm{m}^{3 / 2}\right)$ as the proportional constant to give Equation $\left(8_{\mathrm{v}}\right)$ and by its multiplication of both sides with the $W_{\text {applied }} / W_{\text {indent }}$ ratio $=$ 1.25 that is herewith also deduced. Equation $\left(8_{v}\right)$ is used when only the volume formation must be considered as e.g. in Section 5. Equation (1) thus describes the whole indentation.

$$
\begin{gathered}
F_{N}=F_{N v}^{m} F_{N p}^{n} \\
V_{\text {cone }}=h^{3} \pi \tan \alpha^{2} / 3 \\
F_{N}=F_{N v}^{2 / 3} F_{N p}^{1 / 3} \\
F_{N v}^{2 / 3} \propto h \text { or } F_{N v} \propto h^{2 / 3} \\
F_{N v}=k_{v} h^{3 / 2}
\end{gathered}
$$

Equation (1) is generally valid for all materials with respect to the used cone angle and for pyramids and wedges with their effective cone angles (e.g. $\alpha=$ $70.3^{\circ}$ for the most used Berkovich pyramid). Normalization of these with $\tan \alpha^{2}$ is possible when different indenter geometries among these must be compared. The $k_{v}$ value of Equation $\left(8_{v}\right)$ is the physical hardness with respect to the force $F_{N_{V}}$ that is responsible for the volume formation. The technically more important $k=1.25 k_{v}$ value of Equation (1) is the physical hardness for the whole indentation force with $F_{N}=1.25 F_{N r}$ We thus distinguish two different physical hardness values. This has to be taken into account for the comparison of e.g. pyramidal indentations with spherical ones with different $W_{\text {applied }} / W_{\text {indent }}=F_{N} / F_{N V}$ ratios. Unlike iterations for false "ISO-hardness" we obtain from the correct analysis of Equation (1) by plotting $F_{N}$ vs $h^{3 / 2}$ (the "Kaupp-plot") linear regression lines via Excel $^{(\mathrm{R})}$ calculation and detect the phase-transition onsets at the kink unsteadiness of intersecting regression lines, because different polymorphs exhibit different $k$-values. The calculated intersection point by equalization of the regression lines at $F_{\text {Nkink }}$ and $h_{\text {kink }}^{3 / 2}$ reveals also the practically important indentation energies [cf Equation (2) and Equation (3)]. Furthermore, the phase-transition energy can be calculated by using the Equation (9) through (13). The application Equation (9) and Equation (11) contain the corrections for axis-cut $F_{a}$ when not 
zero. All of these have been deduced and are repeated here in modified form [14] [15] for direct comparison with the spherical situation in Section 2.2.

$$
\begin{gathered}
W_{\text {applied } 1}=0.5 h_{\text {kink }}\left(F_{\text {Nkink }}+F_{a 1}\right) \\
W_{\text {indent } 1}=0.8 W_{\text {applied } 1} \\
W_{\text {indent } 2}=0.4\left(h^{5 / 2}-h_{\text {kink }}^{5 / 2}\right)+F_{a 2}\left(h_{2}-h_{\text {kink }}\right) \\
\text { full } W_{\text {applied }}=0.5 F_{N 2} h_{2} \\
W_{\text {transition }}=\text { full } W_{\text {applied }}-\Sigma W_{\text {applied }}
\end{gathered}
$$

The fast calculation of $W_{\text {applied }}$ for cones, pyramids and wedges with Equation (10) avoids the integration of Equation (1). Higher phase-transition $W_{\text {indents }}$ must be integrated from kink to the next kink etc. The $F_{\mathrm{N}^{2}}-h_{2}$ pair can be freely chosen above the $k_{i n k_{1}}$. In the case of several phase-transitions one proceeds from kink to further kink and after the last phase-transition there is free choice for the $F_{N n}-h_{n}$ pair. A practical example will be calculated in Section 3.

\subsection{The Correct Loading Curve and the Energetics of Spherical Indentations}

A one member "parabola with exponent 3/2" for spheres in [6], [7] and ISO is impossible, because such parabola with exponent $3 / 2$ is only valid for cones, pyramids and wedges. The geometric deduction of the correct $F_{N}$ vs $h$ curve of sphere calottes (with sphere radius $R$ and calotte radius $r$ ) is more involved, because the depth related $R / h$ ratio is changing during the penetration as shown in [10]. As in the conical case one starts again with Equation (4) to distinguish pressure and volume. The sphere-calotte volume formula is $V=h^{2} \pi(R-h / 3)$. It is modified by multiplication with $1=h / h$ to give the more easily handled Equation (14) that is more similar to Equation (5) containing $h^{3}$ but with the varying dimensionless $R / h$ term for its $\pi(R / h-1 / 3)$ correction. This correction term can be treated like a variable factor that has to be separately applied for every force point of the plot, according to Equation (18) that is not at all a one-member parabola. The sequence of the deduction is now similar to the one for cones. We consider again that the total pressure must be proportional to the immersed volume of Equation (14) and get the Formulas (15) for $F_{N}$ (16) for $F_{N p}^{1 / 3}$ and (17) for $F_{N v}^{2 / 3}$. Equation $\left(18_{v}\right)$ is obtained for $F_{N V}$ after multiplication with the material's proportionality factor $k_{s v}$ ( ${ }_{s}$ for sphere; ${ }_{v}$ for volume). Equation $\left(18_{v}\right)$ describes only the volume formation and it is used when the penetration part $F_{N_{V}}$ must be exclusively considered. Equation (18) for the whole indentation is obtained by multiplication of both $F_{N_{V}}$ and $k_{V}$ with the $W_{\text {applied }} / W_{\text {indent }}$ ratio. Equation (18) is used for plotting $F_{N}$ vs $\left\{h^{3 / 2} \pi(R / h-1 / 3)\right\}$. One obtains the penetration resistance values $k_{s 1}$ and $k_{s 2}\left(\mathrm{mN} / \mu \mathrm{m}^{3 / 2}\right)$, the phase-transition onset with $F_{N k i n k}$ the $\left\{h^{3 / 2} \pi(R / h-1 / 3)\right\}$ value, and the $W_{\text {applied }}$ at the kink position from the intersection of the regression lines. The necessary $h_{\text {kink }}$ must not be calculated. It is available from the $F_{N} h$ for $F_{N}=F_{N k i n k}$ curve. For the now necessary calculation of $W_{\text {indent }}$ we reformulate Equation (18) and add the axis cut $F_{a}$ 
$(+, 0$, or -$)$ to give Equation (19). Its integration gives Equation (20) for $W_{\text {indent }}$ The $W_{\text {applied } 2}$ is calculated from $F_{N k i n k}$ to $F_{N 2}$ (here chosen at $F_{N \max }$ ). The balance of full $W_{\text {applied }}-\Sigma W_{\text {applied }}$ is the phase-transition energy $W_{\text {transition }}$ as in Equation (13). A practical example will be calculated in Section 4 . Equation $\left(18_{v}\right)$ will be used in Sections 4, 5, and 6 .

$$
\begin{gathered}
V=h^{3} \pi(R / h-1 / 3) \\
F_{N} \propto h^{3} \pi(R / h-1 / 3) \\
F_{N p}^{1 / 3} \propto h^{1 / 3}[\pi(R / h-1 / 3)]^{1 / 3} \\
F_{N v}^{2 / 3} \propto h^{2 / 3}[\pi(R / h-1 / 3)]^{2 / 3} \\
F_{N v}=k_{s v} h^{3 / 2} \pi(R / h-1 / 3) \\
F_{N}=k_{s} h^{3 / 2} \pi(R / h-1 / 3) \\
F_{N}=k_{s} \pi R h^{1 / 2}-k_{s} \pi h^{3 / 2} / 3+F_{a} \\
W_{\text {indent }}=\int F_{N k i n k} \mathrm{~d} h=2 / 3 \cdot k_{s} \pi R h^{3 / 2}-2 / 15 \cdot k_{s} \pi h^{5 / 2}+\Delta F h
\end{gathered}
$$

\section{The Pyramidal Indentation Calculation of Germanium}

For the numerical exemplification, the published data of the semiconductor germanium are chosen from the literature. This covers pyramidal (Berkovich diamond), spherical (diamond) indentations, and hydrostatic anvil compression. The analysis of the Berkovich indentation onto cubic germanium from [16] according to Equation (1) is depicted in Figure 1 with the inserted regression line

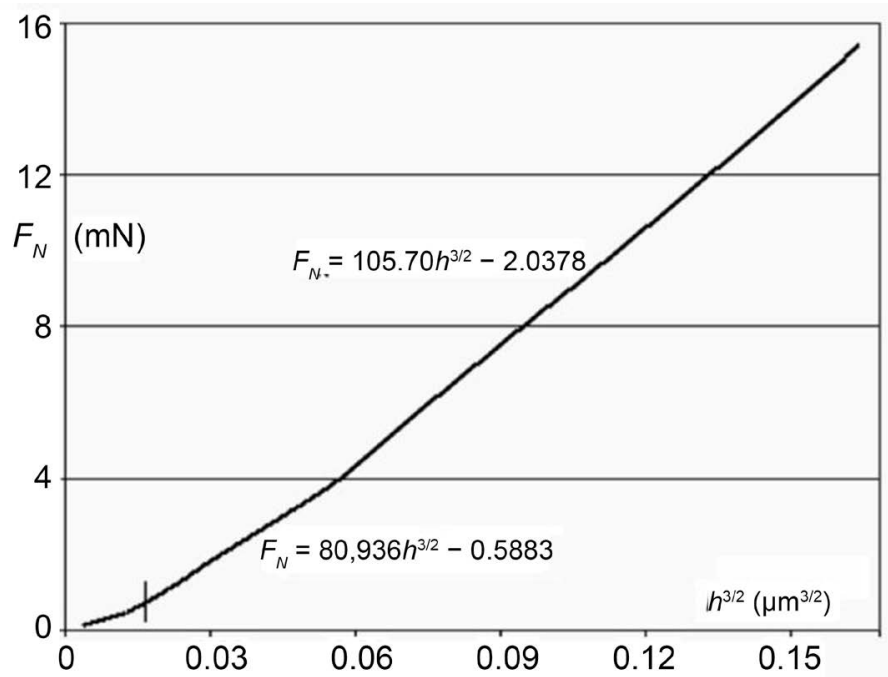

Figure 1. Normal force vs depth ${ }^{3 / 2}$ plot upon indentation onto germanium with inserted regression line equations; the vertical line cuts off the initial surface effect; $F_{\text {Nkink }}$ is at $4.149 \mathrm{mN}$; the original data from the $F_{N}$ vs $h$ curve are taken from Figure 5(a) in [16], but only up to $0.3 \mu \mathrm{m}$ depth (at $15.25 \mathrm{mN}$ ) before the numerous load-unload sequences that detract from the smoothness of the further force-depth curve. 
equations after a short initial surface effect (including the inevitable tip rounding). The proportionality of $W_{\text {indent }}=0.8 W_{\text {applied }}$ (Equation (2) and Equation (3)) for a loading parabola with exponent $3 / 2$ in [12] makes it particularly easy to calculate $W_{\text {indent }}$ for every chosen work so that normalization per force unit provides comparable values for different materials. The phase-transition kink position by equalization of the regression lines is at $4.149 \mathrm{mN}$ and $0.151 \mu \mathrm{m}$. The pristine polymorph withstands a phase-transition up to $0.1342 \mathrm{mN} \mu \mathrm{m}$ applied work and $W_{\text {indent } 1}$ is thus $0.1074 \mathrm{mN} \mu \mathrm{m}$. With $W_{\text {applied2 }}=1.7927$ and full $W_{\text {applied }}=$ $2.3059 \mathrm{mN} \mu \mathrm{m}$ the phase transition energy into the second polymorph calculates easily as full $W_{\text {applied }}-\Sigma W_{\text {applied }}\left(\right.$ Equation (13)) to give $W_{\text {transition }}=0.37898 \mathrm{mN} \mu \mathrm{m}$ from kink to $15.25 \mathrm{mN}$ load. These are after normalization per $\mathrm{mN} 0.03414$ $\mathrm{mN} \mu \mathrm{m} / \mathrm{mN}$. Such transition energies based on physically valid application of geometry and arithmetic calculation rules are not available by any other means. Unfortunately, we could not search for further phase-transitions of germanium, due the smoothness lack by the repeated load-unload sequences at higher loads in [16].

\section{The Spherical Indentation Calculation of Germanium}

The spherical indentation onto germanium at a rate of $7 \mathrm{mN} / \mathrm{s}$ follows Equation (18) and Equation (19), but not Johnson's equation of a one exponent $F_{N^{-}} h^{3 / 2}$ parabola for spheres in [6]. The publication of [17] depicts in its Figure 1(a) the loading curve of crystalline germanium onto (100) from a sphere with radius $R \approx$ $4.2 \mu \mathrm{m}$. This was certainly a good sphere at least up to $4 \mu \mathrm{m}$ depth. The so-called "pop-ins" of the $F_{N}$ vs $h$ curve far away from the phase-transition position are not corrected for, because there is no force hold interruptions and there are no "discontinuities" in the $F_{N}$ vs $h$ curve of [17]. Our trial Kaupp-plot in Figure 2 does not result in a straight line as it should if the "Hertzian analysis" of [17] would be correct.

Only our physically correct two-exponent parabola plot for spherical indentations in Figure 3 gives after the initial surface effect two straight lines with a sharp kink, indicating a phase-transition, even though a phase transition was "excluded" by [17] with Raman spectroscopy after unloading. Only a "pinning of slip bands" and or "multiple discontinuities" by "plastic deformation" were suspected. In Figure 3 we plot the normal force $F_{N}$ vs $\left\{h^{3 / 2} \pi(R / h-1 / 3)\right\}$ for germanium, according to Equation (18). It visualizes the linearity for obtaining the penetration resistance values $k\left(\mathrm{mN} / \mu \mathrm{m}^{3 / 2}\right)$ with respect to the spherical tip with radius $4.2 \mu \mathrm{m}$. The phase-transition onset is clearly seen by the kink and the very different $k$-values of the polymorphs. The regression line results are inserted. The slightly steeper data above the horizontal shut-off line are not included in the regression.

We do not dare to claim a "second kink" at about $46 \mathrm{mN}$ load due to the short penetration length, but did not include the data pairs above $46 \mathrm{mN}$ load in the regression. There is also a risk of spheres' quality at higher depths. The $4.2 \mu \mathrm{m}$ 


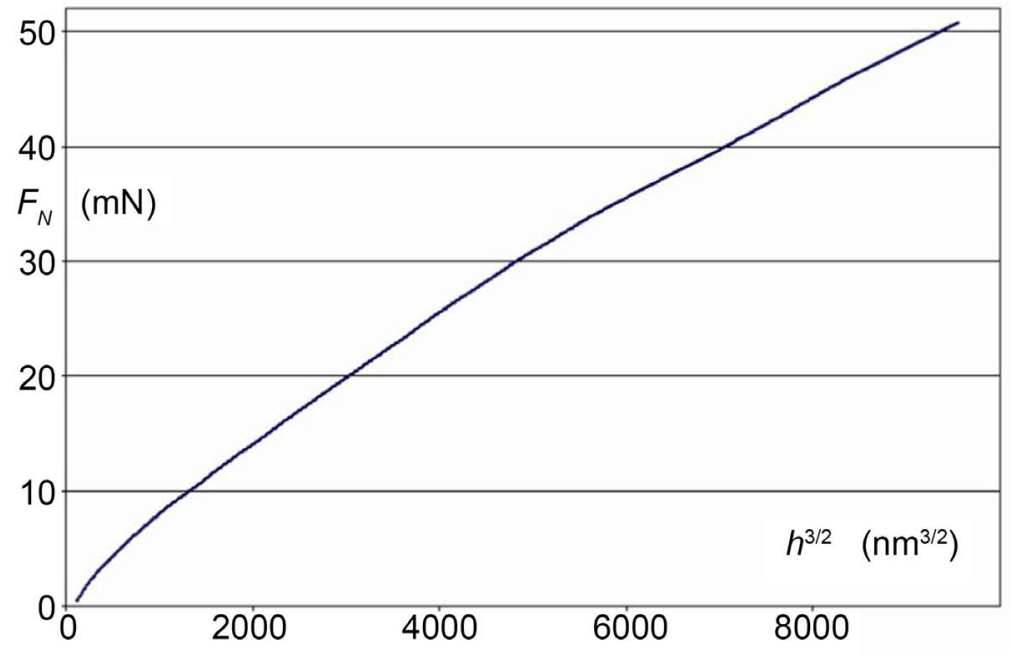

Figure 2. Normal force vs depth ${ }^{3 / 2}$ trial-plot of the spherical $(R=4.2 \mu \mathrm{m})$ indentation onto germanium, disproving the so-called "Hertzian theory" of Johnson [6] that has been claiming a one-exponent $h^{3 / 2}$ parabola as that would require to proceed linearly in that plot; the loading data were taken from [17].

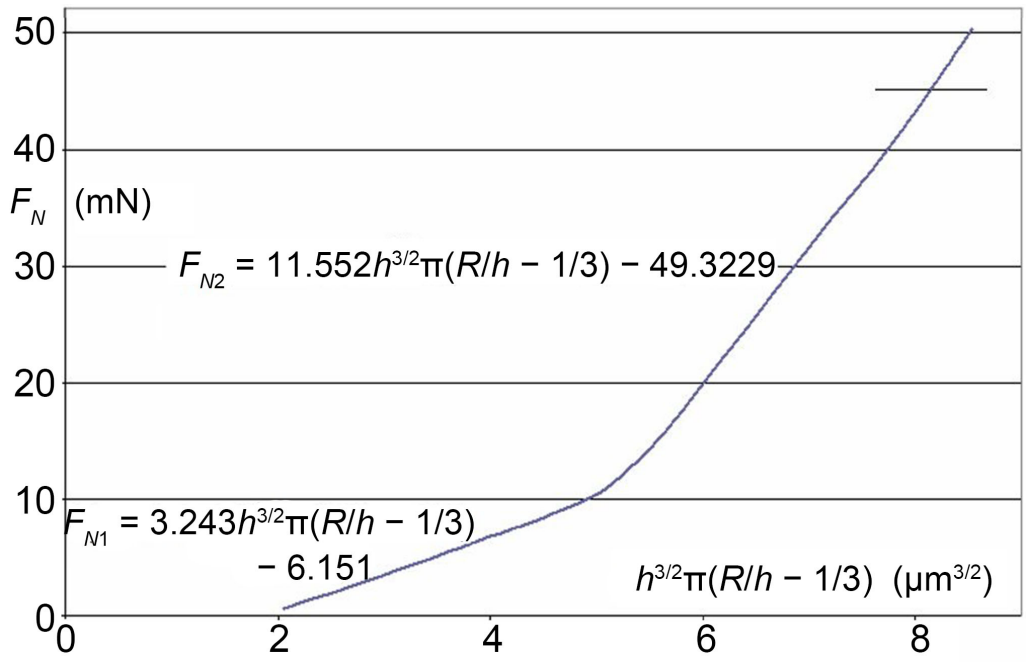

Figure 3. Normal force vs point by point corrected depth ${ }^{3 / 2}$ plot of germanium upon spherical indentation $(R=4.2 \mu \mathrm{m})$ onto germanium with the inserted regression line equations; the initial surface effect data and the data above the horizontal line are not part of the regressions; the phase-transition onset is at $10.703 \mathrm{mN}$; the loading data were taken from [17].

diamond sphere was however in good shape, at least up to about $0.4 \mu \mathrm{m}$ depths. The inserted regression lines give the materials' dependent penetration resistance values $k_{s}\left(\mathrm{mN} / \mathrm{m}^{3 / 2}\right)$ of the respective polymorphs, because the uninterruptedly varying geometric factor is taken care of by the $R / h$ ratios in Equation (18). The equalization of the (not drawn) regression lines provides the sharp phase transition onset at the kink position at $5.197\left\{h^{3 / 2} \pi(R / h-1 / 3)\right\} \mu \mathrm{m}^{3 / 2}$. One obtains $F_{\text {Nkink }}=10.703 \mathrm{mN}$ by insertion in any one of the two regression line equations. With the $F_{\text {Nkink }}$ value one obtains $h_{\text {kink }}=0.124 \mu \mathrm{m}$ from the $F_{N}$ vs $h$ loading 
curve and according to Equation (9) also $W_{\text {applied } 1}=0.67704 \mathrm{mN} \mu \mathrm{m}$. The integrated Equation (20) provides $W_{\text {indentl }}=0.4756 \mathrm{mN} \mu \mathrm{m}$.

The $W_{\text {indent }} / W_{\text {applied }}$ ratio is here not 0.8 as in the case of conical, pyramidal, and wedged indentations (cf Section 3). It changes for every point at spherical indentations as in Figure 3. We need the integrated Formula (20) for the transformed polymorph of germanium. It can be calculated for any force with its depth above the kink value for the calculation of $W_{\text {indent } 2}$ and $W_{\text {applied2 } 2}$. In the absence of a second kink within the loading range we integrated from $F_{N k i n k}$ to $F_{N \max }$ at linearly interpolated $50 \mathrm{mN}$ and the interpolated depth of $h_{\max }=0.4444$ $\mu \mathrm{m}$. Equation (20) provides $W_{\text {indent } 2}=7.07438 \mathrm{mN} \mu \mathrm{m}$ for the sphere with radius $4.2 \mu \mathrm{m}$. Equation (9) is correspondingly used for $\left(50-F_{N k i n k 1}\right)$ and $\left(h_{\max }-h_{\text {kink }}\right)$ to give $W_{\text {applied }}=8.8219 \mathrm{mN} \mu \mathrm{m}$ up to $50 \mathrm{mN}$ load for the sphere of radius 4.2 $\mu \mathrm{m}$. The sum $\left(W_{\text {applied } 1}+W_{\text {applied } 2}\right)$ is 9.4989. As the full applied work (Equation (12)) from 0 to $F_{N \max }$ and $h_{\max }$ is $11.1111 \mathrm{mN} \mu \mathrm{m}$ one obtains the endothermic balance as full $W_{\text {applied }}-\Sigma W_{\text {applied }}=W_{\text {transition }}=1.6122 \mathrm{mN \mu m}$, according to Equation (13) for the phase transition energy of germanium at the kink onset position for the whole indentation force.

It should be noted that the energy calculations for spheres are with respect to the $R / h$ value at the kink position. The non-constancy of the correction factor in Equation (18) prevents a normalization of the energy values per $\mathrm{mN}$. Every energy value must be separately calculated when compared with the values from conical, pyramidal, or wedged indenters. Only for the latter is it possible to interpolate and even interconvert energy values via (effective) cone half angle. This disadvantage of spherical indentations is outweighed by the pressure distribution over an almost plane area. The sphere calotte radius for $R=4.2 \mu \mathrm{m}$ and $h=$ $0.124 \mu \mathrm{m}$ is easily calculated with $\sin \beta=(R-h) / R$ and $\cos \beta=r / R$, to give $r$ $=1.01327 \mu \mathrm{m}$. Thus, the flat $\pi r^{2}$ area is here $3.226 \mu \mathrm{m}^{2}$, which is similar to the calotte-cap $2 \pi R h$ surface area of $3.272 \mu \mathrm{m}^{2}$. For the pressure calculation we need the force part for the penetration of Equation $\left(18_{v}\right)$, in accordance with the energy law. At the phase transition onset position, the $W_{\text {indent1 }} / W_{\text {applied }}$ ratio is $0.4756 / 0.67704=0.70247$. Therefore, also $F_{\text {Nindent } 1} / F_{\text {Napplied } 1}=0.70247$. When this factor is multiplied with the whole force of $10.703 \mathrm{mN}$ one obtains the force at the phase-transformation onset that is only responsible for the penetration and calculates to $7.5185 \mathrm{mN}$. The penetration force/area is thus 2.331 or 2.298 $\mathrm{mN} / \mu \mathrm{m}^{2}$ (which is better known as GPa). We do not decide which of the two surfaces give the better value, but upon rounding both read $2.3 \mathrm{GPa}$. The almost perfect correspondence of this pressure value with much more difficultly obtained hydrostatic anvil pressurization results is discussed in Section 6.

\section{Comparison of the Pyramidal and Spherical Indentations onto Germanium}

It is certainly more precise to indent with diamond indenters like for example with Berkovich indenter, the smooth diamond faces of which are everywhere uniform. Its inevitable tip rounding ends at the very low $h_{\text {cone }}=R(1-\sin 70.3)$ 
and it is mostly hidden within the diverse surface effects that are abandoned. Conversely, ideal spherical diamond tips require more expertise at their production and control of a constant radius for a certain guarantied height. Such radii are not very precisely known and hardly reproducible. The next difference is the penetration mathematics that is very easy for cones, pyramids, and wedges, but more complicated for spheres. These questions could be answered now with the Equation (18) and Equation (19). Different are the penetration depths with pyramids and spheres. The kink values of the phase-transitions for Berkovich are $4.149 \mathrm{mN}$ at $0.151 \cdot 0.8 \mu \mathrm{m}$ (Equation $\left(8_{v}\right)$ ) and for the sphere $10.703 \mathrm{mN}$ at $0.124 \cdot 0.70247 \mu \mathrm{m}$ [Equation $\left(18_{v}\right)$, we must here use the penetration force]. The corresponding $W_{\text {indent } 1}$ values are at $0.10736 \mathrm{mN}$ and $0.4756 \mu \mathrm{m}$ for Berkovich and sphere, respectively. Also, the comparison of the corresponding phase-transition energies of 0.2487 and $1.6217 \mathrm{mN \mu m}$ shows that we cannot claim without further data that these phase-transitions did produce the same polymorph. The Berkovich should have reached a much deeper penetration depth than the sphere and the values of $W_{\text {indent }}$ and $W_{\text {transition }}$ better comparable. It might be twinning of germanium as had been suggested in [16] for the Berkovich. Unfortunately, we could not analyze the smoothness-lacking multi load-unload curves of [16] up to $40 \mathrm{mN}$ so that we probably missed the force for the more demanding phase-transition, as it was reached for the sphere. Furthermore, the lower force for twinning at low depths of the sphere, could have been lost due to zero-point problems at the start and extremely large $R / h$ values of Equation (18) at very low force. This is a disadvantage of spherical indentations. Low energy phase-transitions must be detected with pyramidal indentations. On the other hand, high energy phase-transitions are easier with ideal spheres at lower depths. We detected here two phase-transitions with different transition energies for germanium, the further characterization of these requires X-ray diffraction or more advanced spectroscopic techniques.

\section{Comparison of the Spherical Indentation with Results from Anvil Pressurizing of Germanium}

An important advantage of the sphere calotte geometry is its flat $\pi r^{2}$ area value that is very similar to the one of the $2 \pi R h$ cap area at low depth (here 3.226 and $3.272 \mu \mathrm{m}^{2}$, respectively). One calculates reliable force over area pressure values $\left(\mathrm{mN} / \mu \mathrm{m}^{2}\right.$, better known as GPa). These do not contain the errors of extensive simulations for ISO hardness for indentations in [7] by assuming pristine standards by denying the phase-transitions that had occurred at their loads, not to speak of the numerous further technical errors as revealed and listed in [18]. The present spherical onset force at $\mathrm{F}_{N k i n k}=10.703 \times 0.70247 \mathrm{mN}$ gives 2.321 or 2.298 $\mathrm{mN} / \mu^{2}(\mathrm{GPa})$ transition pressure when divided by the penetration area at the phase-transition onset (Section 4). This pressure value is smaller than those of the most cited anvil experiments that require about 8 to $11 \mathrm{GPa}$, depending on the hydrostatic purity of the pressure transfer in [19]. That seems to exclude in the present spherical case a transformation of Ge-I (cubic, diamond, Fd3m) into 
non-quenchable Ge-II \{(tetragonal, space group I 4(1)/amd, $B$-tin)\}. But one has to consider that Ge-III (body centered tetragonal) is formed from Ge-II in the anvil case upon pressure release from 12 - $14 \mathrm{GPa}$ down to $7.6 \mathrm{GPa}$ and lower, where it thermally reverts to Ge-I and Ge-III. Very important in this respect is the long-time (weeks) anvil-pressurizing of Ge-I at $2.5 \mathrm{GPa}$ to obtain Ge-III at room temperature. This is known since 1965 [20] and has been confirmed in [21]. This value corresponds very well with the rapidly reached phase-transition pressure value of $2.3 \mathrm{GPa}$ by spherical indentation. We conclude that there was enough pressure for the phase-transition under the sphere to yield the Ge-III polymorph. But we cannot exclude that this might have occurred via Ge-II that thermally diverted rapidly to Ge-I and Ge-III at the pressure of $2.3 \mathrm{GPa}$. Both anvil and spherical indentation techniques require $\mathrm{X}$-ray diffraction analyses. Indentation is much easier and probably more precise than anvil pressurizing.

\section{Conclusions}

This paper compares the mathematical descriptions of conical, pyramidal and wretched indentations with the spherical ones and it numerically exemplifies them with literature data from germanium. The geometrically based mathematical deductions result in arithmetic formulas and application equations. Unprecedented applications are developed. The physically correct formulas are up to replace the false formulas of ISO 14577 that rely on false premises that are still violating the energy law by using elastic theory and iterated projected (contact) area. We therefore urgently ask to abandon historical beliefs. ISO 14577 still standardizes incorrect standards and procedures that do not match with reality. The reasons for the inconsistencies for more than a century are unrepeatable extremely complicated "mathematical deductions". In addition to that ISO-ASTM use experimentally false standards not only due to not considering phase-transitions under load-that they cannot detect with their false formulas-, but also with poor force linearities and mix-ups of the standards. It is therefore clear that they could not create valid closed equations for indentations. Rather the false belief required numerous iterations, approximations, data-adjustments, and simulations. Any control of experimental data had been impeded and did not occur in the mainstream. It produced false thinking and encouraged various data manipulations. Some striking examples are challenged in [10] and [18]. These can only now be easily detected and often corrected by using our mathematically correct closed equations. We sincerely advocate to after all accepting the easiest application of geometry and arithmetic calculation rules for the correct analyses of indentation data.

We complete and extend in this paper the geometric solutions for conical, pyramidal, wedged and spherical indentations by physically sound use of the indenter volume for the coupled pressure and penetration events and we deduce valid formulas and application equations. The calculation of the energies and transition energies of phase transitions upon indentation with the Berkovich in- 
denter is calculated in the usual way as in [11] [12] [14] [15] [18] up to $16 \mathrm{mN}$ load. It confirmes the loading curve $F_{N}=k h^{3 / 2}$ from [13].

The still worldwide accepted Johnson equation for spherical indentations (“ $P=4 / 3 E^{*} R^{1 / 2} h^{3 / 2}$ ”) has again been disproved with a trial Kaupp-plot $F_{N}$ vs $h^{3 / 2}$ that is not linear.

The spherical loading curve does not at all proceed as a one exponent parabola, because the volume of the sphere calotte $V=h^{2} \pi(R-h / 3)$ can be transformed into $V=h^{3} \pi(R / h-1 / 3)$ by multiplication with $1=h / h$. In correspondence to the deduction of Equation (4) into Equation (8) [13] one obtains

$F_{N v}=k_{s v} h^{3 / 2} \pi(R / h-1 / 3) \quad\left(18_{v}\right)$ and (18) as the plottable equation with a point by point variable dimensionless correction term. It provides the materials constants $k_{s 1}$ and $k_{s 2}\left(\mathrm{mN} / \mu \mathrm{m}^{3 / 2}\right)$ and the onset values of the phase-transition at the intersection of the regression lines. For the energetic terms one transforms Equation (18) into Equation (19) $F_{N}=k_{s} \pi R h^{1 / 2}-k_{s} \pi h^{3 / 2} / 3+\Delta F$ for the integrations by taking care of their ranges to obtain the indentation energies $W_{\text {indent } 1}$ at the intersection point and $W_{\text {indent } 2}$ at an arbitrary point. The chosen point must be the same for the full applied work (full $W_{\text {applied }}=0.5 F_{N 2} h_{2}$ ). The unprecedented phase-transition energy $W_{\text {transition }}$ is then simply the balance of full $W_{\text {applied }}$ minus $\left(W_{\text {applied } 1}+W_{\text {applied } 2}\right)$.

The unprecedented indentation energy and the phase-transition energy also for spherical indentations enabled the comparison of Berkovich indentations with spherical ones. This was only possible with the penetration forces (not with the full forces from the loading curves!) and thus also with the corresponding $W_{\text {indent } 1}$ values. Only these are comparable and the spherical $W_{\text {applied }} / W_{\text {indent }}$ ratios are depth dependent.

The indentations onto germanium were exemplified and the outcome is different with Berkovich at $4.149 \mathrm{mN}$ load and with $42 \mu \mathrm{m}$ sphere radius at 10.703 $\mathrm{mN}$ load. These are different phase-transitions. The low energy transition (most likely twinning) must have been lost in the spherical case where one detects the Ge-I into Ge-III transition.

Importantly, the spherical indentation reveals a reliable pressure calculation for the comparison with published hydrostatic anvil results, because the flat calotte surface at the low penetration depths is only slightly smaller than the cap surface (here 1.4\%). The average calculated force/ $\mu \mathrm{m}^{2}$ value of the phase-transition pressure amounts to $2.3 \mathrm{mN} / \mu \mathrm{m}^{2}$ (GPa) in excellent correspondence with the published anvil value of $2.5 \mathrm{GPa}$. These results support the interpretation of the hydrostatic anvil results. Such now possible comparisons are very rewarding.

The presented geometric results are not only comprehensive for academia, but the search for phase-transition onset and energy under load is of immense importance for practical applications and safety, because polymorph interfaces are prominent sites of cracking and crashing as imaged in [14]. False historical science must urgently be abandoned for the sake of sound mathematics with undeniable calculation rules. This helps in minimizing the risk for catastrophic crashes. One must now apply the geometry-based indentation in addition to the 
macroscopic pulling and bending tests. Indentations on the geometric mathematical basis is the only way for detecting materials' phase-transition onsets and energies and temperature-dependent including activation energies [22]. The phase-transition onsets for stressed materials must be well above the highest imaginable stresses when they are at work. The search for them is indispensable. This includes the physical indentation control after long stress exposure terms, because a good phase-transition onset can become worse, as material grain structures can change by various influences. There are certainly liability problems, but it's up now for an urgent revision of the still obligatory ISO 14577 standards that enforce false "State-of-the Art" techniques to certification agencies and from them to the producing industries. That is world-wide required for the sake of daily security, not only for the aviation.

\section{Conflicts of Interest}

The author declares no conflicts of interest regarding the publication of this paper.

\section{References}

[1] Boussinesq, J. (1885) Applications des Potentiels a l'Etitude de l'Equilibre et du Mouvement des Solides Elastiques, Gauthier-Villiars, Imprimeur-Libraire, Paris; Book digitized by Google from the library of the University of Michigan and uploaded to the Internet Archive by user tpb. Openlibrary edition OL20553862M.

[2] Hertz, H. (1882) On the Contact of Rigid Elastic Solids and on Hardness 1882. Macmillan and Co., New York. https://archive.org/details/cu31924012500306

[3] Hertz, H. (1896) On the Contact of Rigid Elastic Solids and on Hardness 1896. Macmillan and Co., New York. https://archive.org/details/cu31924012500306

[4] Love, A.E.H. (1939) Boussinesq's Problem for a Rigid Cone. The Quarterly Journal of Mathematics (Oxford), 10, 161-175. https://doi.org/10.1093/qmath/os-10.1.161

[5] Sneddon, I.N. (1965) The Relation between Load and Penetration in the Axisymmetric Boussinesq Problem for a Punch of Arbitrary Profile. International Journal of Engineering Science, 3, 47-57. https://doi.org/10.1016/0020-7225(65)90019-4

[6] Johnson, K.L. (1985) Contact Mechanics. Cambridge University Press, Cambridge. https://doi.org/10.1017/CBO9781139171731

[7] Oliver, W.C. and Pharr, G.M. (1992) An Improved Technique for Determining Hardness and Elastic Modulus Using Load and Displacement Sensing Indentation Experiments. Journal of Materials Research, 7, 1564-1583. https://doi.org/10.1557/JMR.1992.1564

[8] Sangwal, K., Gorostiza, P., Servat, J. and Sanz, F. (1999) Atomic Force Microscopy Study of Nanoindentation Deformation and Indentation Size Effect in MgO Crystals. Journal of Materials Research, 14, 3973-39829. https://doi.org/10.1557/JMR.1999.0537

[9] Kaupp, G. and Naimi-Jamal, M.R. (2004) Nanoscratching on Surfaces: The Relationships between Lateral Force, Normal Force and Normal Displacement. International Journal of Materials Research, 95, 297-305. https://doi.org/10.3139/146.017952

[10] Kaupp, G. (2019) The Loading Curve of Spherical Indentations Is Not a Parabola 
and Flat Punch Is Linear. Advances in Materials Physics and Chemistry, 9, 141-157. https://doi.org/10.4236/ampc.2019.98012

[11] Kaupp, G. (2020) The Use of Geometry Leads to Easy Mathematical Solutions of Indentations. Advances in Materials Physics and Chemistry, 10, 77-95. https://doi.org/10.4236/ampc.2020.103007

[12] Kaupp, G. (2013) Penetration Resistance: A New Approach to the Energetics of Indentations. Scanning, 35, 392-401. https://doi.org/10.1002/sca.21080

[13] Kaupp, G. (2016) The Physical Foundation of $F_{\mathrm{N}}=k h^{3 / 2}$ for Conical/Pyramidal Indentation Loading Curves. Scanning, 38, 177-179. https://doi.org/10.1002/sca.21223

[14] Kaupp, G. (2018) Six Polymorphs of Sodium Chloride Upon Depth-Sensing Scanning Macroindentation with Unusual Long-Range Cracks Requiring $30 \mathrm{~N}$ Load. Journal of Material Sciences and Engineering, 7, 473-483. https://doi.org/10.4172/2169-0022.1000473

[15] Kaupp, G. (2019) Physical Nanoindentation: From Penetration Resistance to PhaseTransition Energies. Advances in Materials Physics and Chemistry, 9, 103-117. https://doi.org/10.4236/ampc.2019.96009

[16] Gogotsi, Y.G., Domnich, V., Dub, S.N., Kailer, A. and Nickel, K.G. (2000) Cyclic Nanoindentation and Raman Microspectroscopy Study of Phase Transformations in Semiconductors. Journal of Materials Research, 15, 871-879. https://doi.org/10.1557/JMR.2000.0124

[17] Bradby, J.E., Williams, J.S., Wong-Leung, J., Swain, M.V. and Munroe, P. (2002) Nanoindentation-Induced Deformation of Germanium. Applied Physics Letters, 80, 2651-2653. https://doi.org/10.1063/1.1469660

[18] Kaupp, G. (2019) Phase-Transition Energies, New Characterization of Solid Materials and Anisotropy. Advances in Materials Physics and Chemistry, 9, 57-70. https://doi.org/10.4236/ampc.2019.94006

[19] Menoni, C.S., Hu, J.Z. and Spain, I.L. (1986) Germanium at High Pressures. Physical Review B, 34, 362-368. https://doi.org/10.1103/PhysRevB.34.362

[20] Bates, C.H., Dachille, F. and Roy, R. (1965) High-Pressure Transitions of Germanium and New High-Pressure Form of Germanium. Science, 147, 860-862. https://doi.org/10.1126/science.147.3660.860

[21] Nelmes, R.J., McMahon, M.I., Wright, N.G., Allan, D.R. and Loveday, J.S. (1993) Stability and Crystal Structure of BC8 Germanium. Physical Review B, 48, 98839886. https://doi.org/10.1103/PhysRevB.48.9883

[22] Kaupp, G. (2014) Activation Energy of the Low-Load NaCl Transition from Nanoindentation Loading Curves. Scanning, 36, 582-589.

https://doi.org/10.1002/sca.21158 